\title{
Laparoscopic colectomy after transcatheter aortic valve implantation in an elderly patient with obstructive descending colon cancer and severe aortic stenosis: a case report
}

Koki Tamai ${ }^{1}$, Shu Okamura ${ }^{1 *}$, Tomohiro Kitahara ${ }^{1}$, Takayuki Minoji ${ }^{1}$, Hiroyuki Takabatake ${ }^{1}$, Noriyuki Watanabe ${ }^{1}$, Noriyuki Yamamura ${ }^{1}$, Nariaki Fukuchi ${ }^{1}$, Chikara Ebisui ${ }^{1}$, Hideoki Yokouchi ${ }^{1}$, Masaki Tsuda ${ }^{2}$, Isamu Mizote ${ }^{2}$ and Masakatsu Kinuta'

\begin{abstract}
Background: The demand for laparoscopic colectomy is increasing due to greater number of elderly colon cancer patients, and it is important to evaluate existing comorbidities to ensure perioperative safety. Aortic stenosis (AS) is one of the most common heart diseases in the elderly, and elderly cancer patients with severe AS may be considered ineligible for optimal cancer treatment if they cannot endure surgical aortic valve replacement (SAVR). Recently, transcatheter aortic valve implantation (TAVI) has become a valid option in patients who are high risk for SAVR. We herein present the first case of an elderly cancer patient with severe AS who underwent laparoscopic colectomy after TAVI.
\end{abstract}

Case presentation: An 87-year-old woman with a history of multiple cardiovascular diseases was diagnosed with obstructive descending colon cancer and initially underwent colonic stenting. However, as preoperative echocardiography revealed severe AS, she underwent TAVI prior to the colectomy to reduce perioperative risk. TAVI was chosen instead of SAVR due to high SAVR mortality risk, and laparoscopic colectomy was performed 22 days after TAVI. Her postoperative course was uneventful, and she was discharged 14 days later without any deterioration in general condition. No recurrence was observed at more than 1 year, even without adjuvant therapy.

Conclusion: TAVI facilitated subsequent laparoscopic colectomy in an elderly cancer patient with severe AS. Our case report shows that TAVI may enable further cancer treatment even in patients with severe AS, who may otherwise be considered not suitable for such treatments.

Keywords: Laparoscopic colectomy, Severe aortic stenosis, Transcatheter aortic valve implantation

\footnotetext{
*Correspondence: okashu@ba3.so-net.ne.jp

'Department of Surgery, Suita Municipal Hospital, Kishibeshinmachi 5-7,

Suita, Osaka 564-8567, Japan

Full list of author information is available at the end of the article
} 


\section{Background}

Increasing life expectancy has led to a greater number of elderly cancer patients requiring colorectal surgery, and laparoscopic procedures are preferred due to the need for minimally invasive surgery in the elderly. Additionally, careful attention should be paid to the presence of comorbid cardiopulmonary disease because a pneumoperitoneum decreases both cardiac output and pulmonary compliance.

Aortic stenosis (AS) is estimated to be present in 4-6\% of the elderly. Although surgical aortic valve replacement (SAVR) has been the standard treatment in severe AS patients, the patients with advanced age, heart failure, or cancer are sometimes considered ineligible for the procedure due to its high perioperative mortality and morbidity risk. Thus, cancer patients ineligible for SAVR may not be able to undergo sufficient cancer treatment.

Recently, transcatheter aortic valve implantation (TAVI) has become a valid option in patients who are high risk for SAVR. While TAVI has facilitated sufficient and subsequent cancer treatment, little is known on the usefulness of TAVI prior to cancer surgery. Herein, we describe the case of an elderly cancer patient with severe AS who underwent laparoscopic colectomy after TAVI.

\section{Case presentation}

An 87-year-old woman was referred to our hospital in poor general condition (Eastern Cooperative Oncology Group performance status 3) with dyschezia and loss of appetite for the past week. A colonoscopy confirmed an obstructive descending colon cancer that could not be penetrated by the colonoscope (Fig. 1a), and computed tomography revealed an obstructive ileus (Fig. 1b). The patient's medical history included coronary artery bypass surgery 11 years ago, surgery for dissecting aortic aneurysm 8 years ago, pacemaker implantation for sick sinus syndrome a year ago, chronic renal failure, and type 2 diabetes. Although a colectomy to reduce the obstruction was required at the earliest possible time, we first placed a colonic stent on the first day of admission to gain some time for preoperative evaluation to reduce perioperative risk (Fig. 2a, b). Then, preoperative echocardiography on the second day after admission revealed severe AS with an aortic valve area of $0.78 \mathrm{~cm}^{2}$, mean aortic pressure gradient of $48 \mathrm{mmHg}$, a maximum jet velocity of $4.2 \mathrm{~m} / \mathrm{s}$, and an ejection fraction of $63 \%$. Although manifestations of severe AS were not clear due to her reduced activity levels, we thought that treating severe AS prior to the colectomy was not entirely wrong because having symptoms were undeniable, and the brain natriuretic peptide, which is one of the risk factors for complication of AS, was high $(216.1 \mathrm{pg} / \mathrm{mL})$. Furthermore, the high surgical risk for colectomy calculated from the American College of Surgeons National Surgical Improvement Program Surgical risk calculator (20.9\% of serious complication and $5.0 \%$ of mortality risk) was also a reason to prioritize the treatment of AS. It is because the deterioration of the general condition, which was one of the main factors of surgical risk, might be caused by severe AS. However, as SAVR mortality risk was evaluated to be high at $8.7 \%$ (according to the European system for cardiac operative risk evaluation II) and adverse events and surgical stress after SAVR may delay or prevent the colectomy, we proceeded with TAVI instead of SAVR.

The patient underwent TAVI through the right femoral artery on day 31 after colonic stenting. A 26-mm Evolut $\mathrm{R}$ valve was placed under fluoroscopic guidance (Fig. 3) and echocardiography 7 days after the procedure showed an improvement in the mean aortic pressure gradient to $7 \mathrm{mmHg}$ with an aortic valve area of 1.82 $\mathrm{cm}^{2}$ and the ejection fraction to $70 \%$.

To reduce probable tumor bleeding, we administered aspirin alone from TAVI until colectomy, even though dual antiplatelet therapy is recommended. The patient received 10,000 unit/day heparin administration for 7 days instead of aspirin until $5 \mathrm{~h}$ prior to surgery and then underwent laparoscopic colectomy 22 days after TAVI. We ligated left colic artery, which was the main

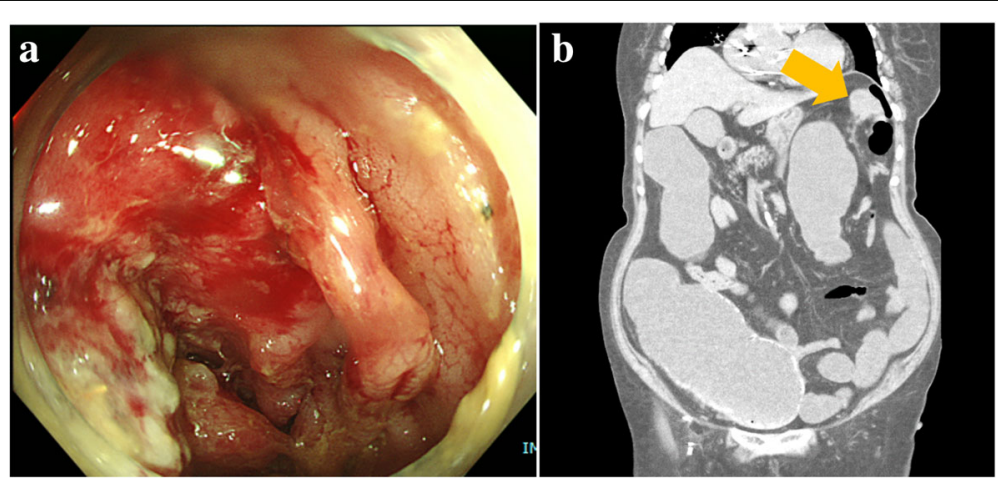

Fig. 1 Preoperative colonoscopy and computed tomography findings. a Colonoscopy confirmed descending colon cancer and obstruction. b Computed tomography revealed an obstructive ileus due to descending colon cancer (yellow arrow) 


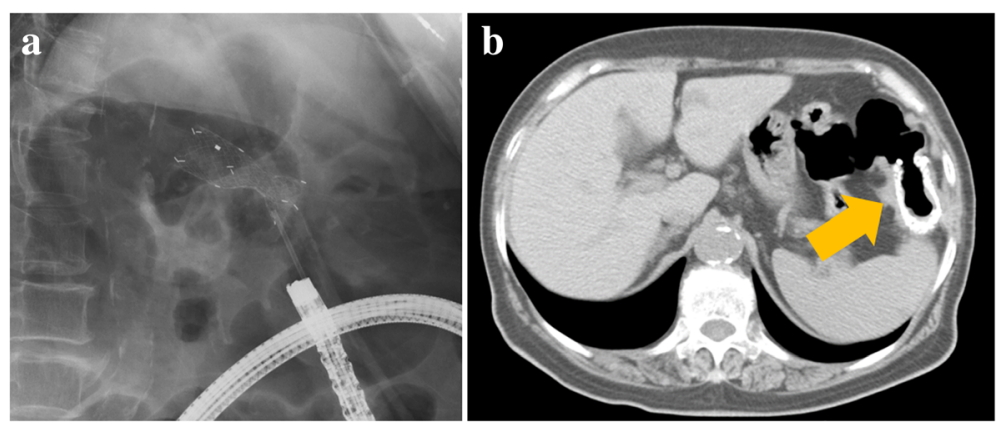

Fig. 2 Fluoroscopy and computed tomography findings after colonic stent placement. a Colonic stent was placed in the descending colon under fluoroscopic guidance. b Colonic stent (yellow arrow) reduced the obstruction

feeding artery of the tumor, and performed D3 lymph node dissection using a 5 -port technique in the supine position. The colon was resected with sufficient distal and proximal margins from the tumor and anastomosed. The operation time was $346 \mathrm{~min}$ and the estimated blood loss was $10 \mathrm{ml}$. Pathologically, the tumor was diagnosed as T4a, N1b (3/32), M0, Stage IIIB. Her postoperative course was unremarkable, and she was discharged 14 days after colectomy. No recurrence was observed for more than 1 year without adjuvant therapy.

\section{Discussion}

Laparoscopic surgery for colorectal cancer is a minimally invasive procedure that is associated with less postoperative pain, fewer complications, faster recovery, and greater early social rehabilitation $[1,2]$. Therefore, it is suitable for high-risk patients, especially those with frailty and in the elderly. In recent years, laparoscopic

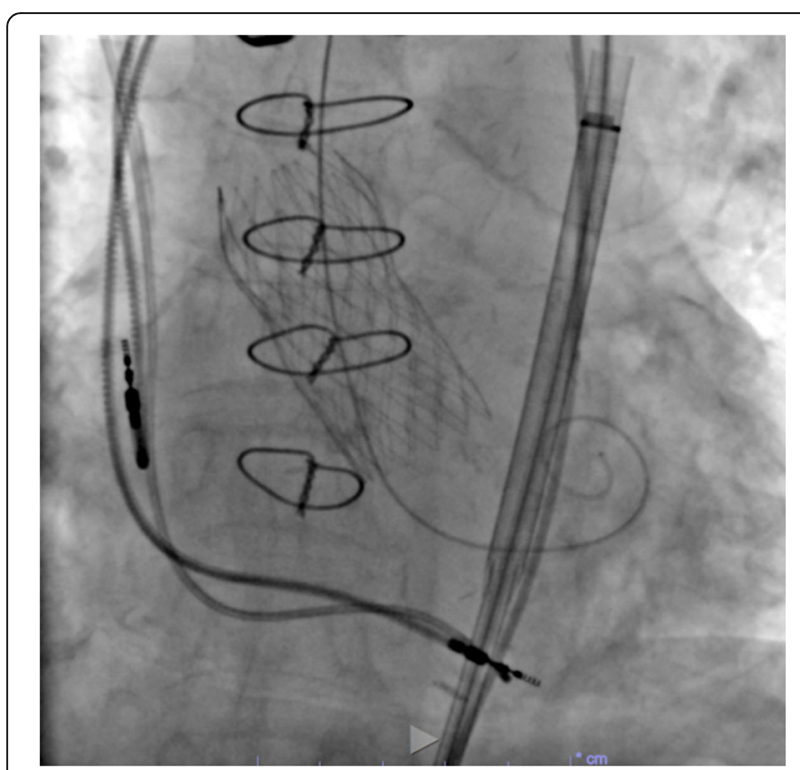

Fig. 3 Fluoroscopy during transcatheter aortic valve implantation with a self-expanding device (26-mm Evolut $\mathrm{R}$ valve) surgery in such high-risk populations has been reported to have fewer complications and lower mortality rates compared with open laparotomy [3-5]. Meanwhile, in patients with low cardiac or pulmonary function, a pneumoperitoneum would exacerbate cardiopulmonary function by decreasing cardiac output and pulmonary compliance [6]. Therefore, it is essential to evaluate cardiopulmonary function prior to laparoscopic surgery. Our patient had a history of multiple cardiovascular diseases, and we performed echocardiography to evaluate her current cardiovascular status, which incidentally revealed severe AS. Symptoms of AS such as shortness of breath and palpitation at the time of exertion may not be readily apparent in the elderly with reduced activity, and in cancer patients, anemia and fatigue caused by the disease itself can mask the presence of cardiopulmonary symptoms. Our case serves to confirm the importance of careful preoperative evaluation, especially in elderly cancer patients.

AS is a narrowing of the aortic valve opening that leads to the obstruction of the left ventricular outflow, subsequent left ventricular systolic dysfunction, and ultimately heart failure in the long-term. In patients undergoing non-cardiac surgery, severe AS is a known high-risk factor of mortality and morbidity because it reduces coronary perfusion under surgical stress and hemodynamic changes. The European Society of Cardiology guidelines recommend SAVR in symptomatic severe AS patients prior to non-cardiac surgery [7], while in asymptomatic severe AS patients, SAVR is recommended only when the risk of non-cardiac surgery is high, and the risk of SAVR is low. Our patient did not meet the absolute indications for SAVR because of her poor symptoms; however, we thought it was better to prioritize the treatment of AS using TAVI over colectomy because AS severity and symptoms, which can increase the risk of colectomy, may not be apparent in such an elderly cancer patient.

TAVI is a minimally invasive approach for replacing the aortic valve that differs from SAVR in that it requires 
no cardiac arrest, extracorporeal circulation, or thoracotomy. In high-risk or ineligible patients, the PARTNER trial showed that treatment outcomes, including mortality rates, LV function recovery, and morbidity after TAVI were either comparable to or superior to those of conventional therapy [8-10]. Therefore, TAVI has become the standard alternative treatment in high-risk surgical patients. Furthermore, specific advantages of TAVI have been reported in cancer patients, as it does not require cardiopulmonary bypass (CPB). These include a possible reduction in the risks associated with tumor bleeding due to anticoagulant disorders or anticoagulants administration, and tumor dissemination due to immunosuppressive and inflammatory effects of CPB $[11,12]$. In patients with severe AS requiring cancer treatment, TAVI not only enables a smooth transition to the next treatment strategy due to its minimally invasive nature but also confers an oncological advantage. Thus, TAVI was considered most suitable for our patient. Importantly, our patient felt an improvement in her physical condition after TAVI even though there were no obvious symptoms, and our case serves to highlight the fact that, in addition to careful preoperative evaluation, it is also necessary to make prudent decisions on treatment strategy in elderly patients whose symptoms are often less apparent.

We prescribed a single drug antiplatelet regimen with aspirin alone after TAVI, and laparoscopic colectomy was performed 22 days after TAVI with perioperative heparin bridging. We selected this treatment regimen, even though dual antiplatelet therapy using clopidogrel and aspirin is recommended for the first 6 months after TAVI, to avoid ischemic complications, because of the higher risk of tumor bleeding in our patient. Recent reports have shown that, compared with dual antiplatelet therapy, aspirin alone could reduce the risk of lifethreatening/major bleeding while not increasing the risk of ischemic events following TAVI [13]. Although the preventive effects of aspirin alone in cancer patients with thrombotic tendencies remain unknown, we treated our patient with aspirin alone for fear of tumor bleeding. It is possible that heparin bridging may not have been necessary because of its unclear benefit, and one metaanalysis has shown that antiplatelet therapy at the time of non-cardiac surgery is associated with minimal bleeding risk [14]. Thus, it is possible that the colectomy could have been performed earlier in the absence of heparin bridging.

Due to factors such as age, heart failure, and cancer, $30-50 \%$ of symptomatic AS patients are considered ineligible for SAVR [15-17]. Previously, elderly cancer patients with severe AS similar to our patient may have been forced to forego treatment of severe AS and cancer, but TAVI can potentially help these patients. To the best of our knowledge, this is the first report of a patient undergoing laparoscopic colectomy for cancer after TAVI even though a few surgical reports of cancer surgery after TAVI are available [18-20]. There is no evidence on the feasibility of TAVI in severe AS patients scheduled for cancer surgery and its effects on subsequent surgery. Further studies are required to assess variables such as adequate treatment interval between TAVI and cancer surgery, a perioperative antiplatelet agent, and surgical indications.

\section{Conclusion}

TAVI facilitated a subsequent laparoscopic colectomy in an elderly cancer patient with severe AS. Careful preoperative examination, combined with less invasive procedures, such as TAVI and laparoscopic colectomy, enabled our patient to be treated without any deterioration in general condition. TAVI may increase treatment options in cancer patients considered ineligible for certain therapeutic strategies due to severe AS.

\section{Abbreviations}

AS: Aortic stenosis; CPB: Cardiopulmonary bypass; SAVR: Surgical aortic valve replacement; TAVI: Transcatheter aortic valve implantation

\section{Acknowledgments \\ Not applicable.}

\section{Authors' contributions}

$\mathrm{KT}$ drafted the manuscript and provided the original pictures. SO had revised the manuscript critically. All authors read and approved the final manuscript.

\section{Funding}

The authors declare that this study was not funded externally.

\section{Availability of data and materials}

The authors declare that all data in this article are available within the article.

Ethics approval and consent to participate

Not applicable.

\section{Consent for publication}

Written informed consent was obtained from the patient for publication of this case report and any accompanying images.

\section{Competing interests}

The authors declare that they have no competing interests.

\section{Author details}

${ }^{1}$ Department of Surgery, Suita Municipal Hospital, Kishibeshinmachi 5-7, Suita, Osaka 564-8567, Japan. ${ }^{2}$ Department of Cardiovascular Medicine, Osaka University Graduate School of Medicine, Yamadaoka 2-2, Suita, Osaka 565-0871, Japan.

Received: 19 April 2019 Accepted: 12 June 2019

Published online: 24 June 2019

\section{References}

1. Guillou PJ, Quirke P, Thorpe H, Walker J, Jayne DG, Smith AM, et al. Shortterm endpoints of conventional versus laparoscopic-assisted surgery in patients with colorectal cancer (MRC CLASICC trial): multicentre, randomised controlled trial. Lancet. 2005;365(9472):1718-26.

2. van der Pas MH, Haglind E, Cuesta MA, Fürst A, Lacy AM, Hop WC, et al. Laparoscopic versus open surgery for rectal cancer (COLOR II): short-term outcomes of a randomised, phase 3 trial. Lancet Oncol. 2013;14(3):210-8. 
3. Kothari P, Congiusta DV, Merchant AM. Laparoscopic versus open colectomy: the impact of frailty on outcomes. Updates Surg. 2018. https:/ doi.org/10.1007/s13304-018-0531-7.

4. Rossi BWP, Labib P, Ewers E, Leong S, Coleman M, Smolarek S. Long-term results after elective laparoscopic surgery for colorectal cancer in octogenarians. Surg Endosc. 2019. https://doi.org/10.1007/s00464-019-06747-5.

5. Kannan U, Reddy VS, Mukerji AN, Parithivel VS, Shah AK, Gilchrist BF, et al. Laparoscopic vs open partial colectomy in elderly patients: Insights from the American College of Surgeons - National Surgical Quality Improvement Program database. World J Gastroenterol. 2015;21(45):12843-50.

6. Neudecker J, Sauerland S, Neugebauer E, Bergamaschi R, Bonjer HJ, Cuschieri A, et al. The European Association for Endoscopic Surgery clinical practice guideline on the pneumoperitoneum for laparoscopic surgery. Surg Endosc. 2002;16(7):1121-43.

7. Kristensen SD, Knuuti J, Saraste A, Anker S, Botker HE, Hert SD, et al. 2014 ESC/ESA guidelines on non-cardiac surgery: cardiovascular assessment and management: the joint task force on non-cardiac surgeny: cardiovascular assessment and management of the European Society of Cardiology (ESC) and the European Society of Anaesthesiology (ESA). Eur Heart J. 2014;35(35):2383-431.

8. Elmariah S, Palacios IF, McAndrew T, Hueter I, Inglessis I, Baker JN, et al. Outcomes of transcatheter and surgical aortic valve replacement in highrisk patients with aortic stenosis and left ventricular dysfunction: results from the Placement of Aortic Transcatheter Valves (PARTNER) trial (cohort A). Circ Cardiovasc Interv. 2013;6(6):604-14.

9. Mack MJ, Leon MB, Smith CR, Miller DC, Moses JW, Tuzcu EM, et al. 5-year outcomes of transcatheter aortic valve replacement or surgical aortic valve replacement for high surgical risk patients with aortic stenosis (PARTNER1): a randomised controlled trial. Lancet. 2015;385(9986):2477-84.

10. Kapadia SR, Leon MB, Makkar RR, Tuzcu EM, Svensson LG, Kodali S, et al. 5year outcomes of transcatheter aortic valve replacement compared with standard treatment for patients with inoperable aortic stenosis (PARTNER 1): A randomised controlled trial. Lancet. 2015;385(9986):2485-91.

11. Scott BH, Seifert FC, Glass PS, Grimson R. Blood use in patients undergoing coronary artery bypass surgery: impact of cardiopulmonary bypass pump, hematocrit, gender, age, and body weight. Anesth Analg. 2003;97(4):958-63 table of contents.

12. Pinto CA, Marcella S, August DA, Holland B, Kostis JB, Demissie K. Cardiopulmonary bypass has a modest association with cancer progression: a retrospective cohort study. BMC Cancer. 2013:13:519.

13. Rodes-Cabau J, Masson JB, Welsh RC, Garcia Del Blanco B, Pelletier M, Webb $J \mathrm{G}$, et al. Aspirin versus aspirin plus clopidogrel as antithrombotic treatment following transcatheter aortic valve replacement with a balloon-expandable valve: the ARTE (Aspirin Versus Aspirin + ClopidogRel Following Transcatheter Aortic Valve Implantation) randomized clinical trial. JACC Cardiovasc Interv. 2017;10(13):1357-65.

14. Columbo JA, Lambour AJ, Sundling RA, Chauhan NB, Bessen SY, Linshaw $\mathrm{DL}$, et al. A Meta-analysis of the impact of aspirin, clopidogrel, and dual antiplatelet therapy on bleeding complications in noncardiac surgery. Ann Surg. 2018;267(1):1-10

15. Schechter M, Balanescu DV, Donisan T, Dayah TJ, Kar B, Gregoric I, et al. An update on the management and outcomes of cancer patients with severe aortic stenosis. Catheter Cardiovasc Interv. 2018. https:/doi.org/10.1002/ccd.28052.

16. Thaden JJ, Nkomo VT, Enriquez-Sarano M. The global burden of aortic stenosis. Prog Cardiovasc Dis. 2014;56(6):565-71.

17. lung B, Vahanian A. Epidemiology of acquired valvular heart disease. Can $J$ Cardiol. 2014;30(9):962-70.

18. Sakai T, Yahagi K, Miura S, Hoshino T, Yokota T, Tanabe K, et al. Transcatheter aortic valve implantation for patients with lung cancer and aortic valve stenosis. J Thorac Dis. 2018;10(5):E387-90.

19. Tanaka T, Yahagi K, Okuno T, Horiuchi Y, Kusuhara T, Yokozuka M, et al. Transcatheter aortic valve implantation in a patient with severe aortic valve stenosis, colon cancer, and obstructive ileus: a case report. J Cardiol Cases. 2018;17(5):163-6.

20. Okuno T, Yahagi K, Horiuchi Y, Sato Y, Tanaka T, Koseki K, et al. The role of transcatheter aortic valve replacement in the patients with severe aortic stenosis requiring major non-cardiac surgery. Cardiovasc Interv Ther. 2019. https://doi.org/10.1007/s12928-019-00575-z.

\section{Publisher's Note}

Springer Nature remains neutral with regard to jurisdictional claims in published maps and institutional affiliations.

\section{Submit your manuscript to a SpringerOpen ${ }^{\circ}$ journal and benefit from:}

- Convenient online submission

- Rigorous peer review

- Open access: articles freely available online

- High visibility within the field

- Retaining the copyright to your article

Submit your next manuscript at $\boldsymbol{\nabla}$ springeropen.com 